\title{
Boundary Value Problems in weighted Edge Spaces
}

\author{
G. Harutyunyan ${ }^{1} \quad$ B.-W.Schulze ${ }^{2}$
}

\begin{abstract}
We study elliptic boundary value problems in a wedge with additional edge conditions of trace and potential type. We compute the (difference of the) number of such conditions in terms of the Fredholm index of the principal edge symbol. The task will be reduced to the case of special opening angles, together with a homotopy argument.
\end{abstract}

Keywords: Elliptic operators in domains with edges, boundary value problems, weighted edge spaces.

2000 AMS-classification: 35J40, 47G30, 58J32

\section{Contents}

\section{Introduction $\quad 2$}

1 Tools from the edge calculus $\quad 2$

1.1 The edge formulation of boundary value problems . . . . . 2

1.2 Weighted spaces on infinite cones and principal edge symbols 4

1.3 Weighted edge spaces and symbols with twisted homogeneity 7

2 Fredholm property and index of edge symbols 10

2.1 Ellipticity on the infinite cone . . . . . . . . . . . . . 10

2.2 The index of edge symbols . . . . . . . . . . . . 12

\footnotetext{
${ }^{1}$ State University of Yerevan, Faculty of Informatics and Applied Mathematics, Alex Manukian 1, 375049 Yerevan, Armenia E-mail: gohar@ysu.am

${ }^{2}$ Institut FÜr Mathematik, Universität Potsdam, Postfach 601553, 14415 POTSDAM, GERMANY E-mail: schulze@math.uni-potsdam.de
} 
3 Elliptic edge conditions and parametrices $\quad 17$

3.1 Elliptic edge conditions . . . . . . . . . . . . . 17

3.2 Parametrices in weighted edge spaces . . . . . . . . . . . . . 19

3.3 Concluding remarks . . . . . . . . . . . . . . . . . . . 19

$\begin{array}{ll}\text { References } & 22\end{array}$

\section{Introduction}

Boundary value problems for elliptic differential operators on manifolds with conical points or edges can be studied from the point of view of solvability in weighted Sobolev spaces. Numerous authors have contributed to this subject, see, Kondratyev [10], Kondratyev and Oleynik [11], Maz'ja and Rossmann [12], or the bibliography of [9]. The present paper is aimed at studying a number of specific boundary value problems for the Laplacian in a wedge, under mixed elliptic conditions, and from the point of view of ellipticity with respect to a principal symbolic hierarchy, which is a triple $\left(\sigma_{\psi}(\cdot), \sigma_{\partial}(\cdot), \sigma_{\wedge}(\cdot)\right)$ consisting of interior, boundary and edge symbols. The main issue here is to characterise those weights $\gamma \in \mathbb{R}$ such that the problem in question, interpreted as an operator in scales of weighted edge spaces, admits extra edge conditions and has a parametrix in a corresponding pseudo-differential edge algebra. In particular, we compute the index of the Fredholm family $\sigma_{\wedge}(\mathcal{A})$ which is responsible for the difference of the number of edge conditions of trace and potential type.

We employ the edge calculus of [14], here in the variant of boundary value problems with the transmission property at the smooth part of the boundary, and with a new (compared with [14]) scale of weighted edge spaces, suggested by Airapetyan and Witt [1], and Tarkhanov [18]. For the computation of the index of edge symbols we employ the results of the author's joint paper with Dines [2] and of [5].

\section{Tools from the edge calculus}

\subsection{The edge formulation of boundary value problems}

Consider a differential operator

$$
\tilde{A}=\sum_{|\tilde{\alpha}| \leq \mu} c_{\tilde{\alpha}}(x, y) D_{x, y}^{\tilde{\alpha}}
$$

in $\mathbb{R}_{x}^{2} \times \mathbb{R}_{y}^{q}$ with coefficients $c_{\tilde{\alpha}} \in C^{\infty}\left(\mathbb{R}^{2} \times \mathbb{R}^{q}\right)$, and restrict $A$ to a wedge $K_{\alpha} \times \mathbb{R}^{q}$

$$
K_{\alpha}:=\left\{r e^{i \phi}: r \in \mathbb{R}_{+}, 0 \leq \phi \leq \alpha\right\}
$$


for some $0<\alpha \leq 2 \pi$, where $(r, \phi) \in \mathbb{R}_{+} \times S^{1}$ are polar coordinates in $\mathbb{R}^{2} \backslash\{0\}$. In the case $\alpha=2 \pi$ we distinquish the components $\left\{(r, 0): r \in \mathbb{R}_{+}\right\}$and $\left\{(r, 2 \pi): r \in \mathbb{R}_{+}\right\}$of the boundary, i.e., $K_{2 \pi}$ consists of the set $\mathbb{R}^{2} \backslash\{0\}$, slit along $\mathbb{R}_{x_{1},+}$, where $\partial K_{2 \pi}$ consists of two components of $\mathbb{R}_{x_{1},+}$. The operator $\tilde{A}$ takes the form of an edge-degenerate operator

$$
A=r^{-\mu} \sum_{j+|\beta| \leq \mu} a_{j \beta}(r, y)\left(-r \frac{\partial}{\partial r}\right)^{j}\left(r D_{y}\right)^{\alpha}
$$

with coefficients $a_{j \beta}(r, y) \in C^{\infty}\left(\overline{\mathbb{R}}_{+} \times \mathbb{R}^{q}, \operatorname{Diff}^{\mu-(j+|\beta|)}\left(I_{\alpha}\right)\right), I_{\alpha}:=[0, \alpha]$, where $\operatorname{Diff}^{\nu}\left(I_{\alpha}\right)$ denotes the space of all differential operators of order $\nu$ on $I_{\alpha}$ with smooth coefficients up to the end points. We are mainly interested in the Laplacian

$$
\tilde{A}=\Delta:=\sum_{j=1}^{2} \frac{\partial^{2}}{\partial x_{j}{ }^{2}}+\Delta_{\mathbb{R}^{q}}
$$

for $\Delta_{\mathbb{R}^{q}}:=\sum_{l=1}^{q} \frac{\partial^{2}}{\partial y_{l}^{2}}$ where $\mu=2$ and

$$
A=r^{-2}\left\{\left(r \frac{\partial}{\partial r}\right)^{2}+\partial_{\phi}^{2}+r^{2} \Delta_{\mathbb{R}^{q}}\right\} .
$$

Concerning the boundary conditions at $I_{ \pm} \times \mathbb{R}^{q}$ with $I_{ \pm}:=\left\{\alpha_{ \pm}\right\} \times \mathbb{R}_{+}$, for $\alpha_{-}:=0, \alpha_{+}:=\alpha$ we first consider operators

$$
B_{ \pm}:=r^{-\mu_{ \pm}} \sum_{j+|\beta| \leq \mu_{ \pm}} b_{ \pm, j \beta}(r, y)\left(-r \frac{\partial}{\partial r}\right)^{j}\left(r D_{y}\right)^{\beta}
$$

with coefficients $b_{ \pm, j \beta}(r, y) \in C^{\infty}\left(\overline{\mathbb{R}}_{+} \times \mathbb{R}^{q}, \operatorname{Diff}^{\mu_{ \pm}-(j+|\beta|)}\left(I_{\alpha}\right)\right)$ and set

$$
T_{ \pm}:=\mathrm{r}_{ \pm} B_{ \pm}
$$

where $\mathrm{r}_{ \pm}$means the restriction of a distribution in $H_{\mathrm{loc}}^{s}\left(\operatorname{int} K_{\alpha}\right)$ to $I_{ \pm} \times$ $\mathbb{R}^{q}, s>\frac{1}{2}$. To illustrate our approach we will assume that $B_{ \pm}$have constant coefficients.

The operators

$$
T_{ \pm}:=\mathrm{r}_{ \pm}
$$

represent Dirichlet conditions,

$$
T_{ \pm}:=\mathrm{r}_{ \pm} r^{-1} \partial_{\phi}
$$

Neumann, and

$$
T_{-}:=\mathrm{r}_{-}, \quad T_{+}:=\mathrm{r}_{+} r^{-1} \partial_{\phi}
$$

(or $T_{-}:=\mathrm{r}_{-} r^{-1} \partial_{\phi}, T_{+}:=\mathrm{r}_{+}$) Zaremba conditions. The boundary value problem

$$
A u=f \text { in } \operatorname{int} K_{\alpha} \times \mathbb{R}^{q}, \quad T_{ \pm} u=g_{ \pm} \text {on } I_{ \pm} \times \mathbb{R}^{q}
$$


will be identified with a column matrix of operators $\mathcal{A}:={ }^{\mathrm{t}}\left(\begin{array}{lll}A & T_{-} & T_{+}\end{array}\right)$. Incidentally we also write $T:={ }^{\mathrm{t}}\left(T_{-} \quad T_{+}\right)$and $\mathcal{A}={ }^{\mathrm{t}}\left(\begin{array}{ll}A & T\end{array}\right)$. Moreover, instead of $K_{\alpha}$ we mainly consider the stretched cone $\mathbb{R}_{+} \times I_{\alpha}=: I_{\alpha}^{\wedge}$.

The principal interior symbol $\sigma_{\psi}(\mathcal{A})$ is nothing other than the homogeneous principal symbol $\sigma_{\psi}(A)$ of the operator $A$. For (1) we obtain

$$
\sigma_{\psi}(A)(\rho, \vartheta, \eta)=r^{-2}\left((-i r \rho)^{2}-|\vartheta|^{2}-r^{2}|\eta|^{2}\right),
$$

where $(\rho, \vartheta, \eta)$ denote the covariables of $(r, \phi, y) \in I_{\alpha}^{\wedge} \times \mathbb{R}^{q}$. Observe that

$$
\tilde{\sigma}_{\psi}(A)(\rho, \vartheta, \eta):=r^{2} \sigma_{\psi}(A)\left(r^{-1} \rho, \vartheta, r^{-1} \eta\right)
$$

is smooth up to $r=0$. The principal boundary symbol of $\mathcal{A}$ is defined by

$$
\sigma_{\partial}(\mathcal{A}):={ }^{\mathrm{t}}\left(\sigma_{\partial}(A) \quad \sigma_{\partial}(T)\right)
$$

for $\sigma_{\partial}(T):={ }^{\mathrm{t}}\left(\sigma_{\partial}\left(T_{-}\right) \quad \sigma_{\partial}\left(T_{+}\right)\right)$, where

$$
\begin{gathered}
\sigma_{\partial}(A)(\rho, \eta):=r^{-2} \sigma_{\psi}(A)\left(\rho, D_{\phi}, \eta\right): H^{s}\left(\mathbb{R}_{+}\right) \rightarrow H^{s-2}\left(\mathbb{R}_{+}\right), \\
\sigma_{\partial}\left(T_{ \pm}\right)(\rho, \eta):=\mathrm{r}_{ \pm} r^{-\mu_{ \pm}} \sigma_{\psi}\left(B_{ \pm}\right)(\rho, \eta): H^{s}\left(\mathbb{R}_{+}\right) \rightarrow \mathbb{C}
\end{gathered}
$$

$(\rho, \eta) \neq 0$; here $H^{s}\left(\mathbb{R}_{+}\right):=\left.H^{s}(\mathbb{R})\right|_{\mathbb{R}_{+}}$with the standard Sobolev space $H^{s}(\mathbb{R})$ of smoothness $s \in \mathbb{R}$, and $\sigma_{\psi}\left(B_{ \pm}\right)$are the homogeneous principal symbols of the operators $B_{ \pm}$of order $\mu_{ \pm}$. The operator family

$$
\begin{aligned}
\tilde{\sigma}_{\partial}(\mathcal{A})(\rho, \eta):= & { }^{\mathrm{t}}\left(r^{2} \sigma_{\partial}(A)\left(r^{-1} \rho, r^{-1} \eta\right) \quad r^{\mu_{-}} \sigma_{\partial}\left(T_{-}\right)\left(r^{-1} \rho, r^{-1} \eta\right)\right. \\
& \left.{ }^{\mu_{+}} \sigma_{\partial}\left(T_{+}\right)\left(r^{-1} \rho, r^{-1} \eta\right)\right)
\end{aligned}
$$

is smooth up to $r=0$. In order to explain the so-called principal edge symbol we introduce some weighted function spaces.

\subsection{Weighted spaces on infinite cones and principal edge symbols}

Let us first recall some weighted spaces on $\mathbb{R}_{+}$. Consider the Mellin transform $M u(w):=\int_{0}^{\infty} r^{w-1} u(r) d r$, first on $u \in C_{0}^{\infty}\left(\mathbb{R}_{+}\right)$, where $M u(w)$ is an entire function in $w \in \mathbb{C}$, and then extented to more general classes of distributions (also with values in several kinds of Fréchet spaces). Then the Mellin covariable $w$ will often range on a weight line $\Gamma_{\frac{1}{2}-\gamma}, \gamma \in \mathbb{R}, \Gamma_{\beta}:=\{w \in \mathbb{C}: \operatorname{Re} w=$ $\beta\}$. Recall that the weighted Mellin transform $M_{\gamma}:\left.C_{0}^{\infty}\left(\mathbb{R}_{+}\right) \rightarrow M u\right|_{\Gamma_{\frac{1}{2}-\gamma}}$ extends to an isomorphism $M_{\gamma}: r^{\gamma} L^{2}\left(\mathbb{R}_{+}\right) \rightarrow L^{2}\left(\Gamma_{\frac{1}{2}-\gamma}\right)$ (here function and distribution spaces on $\Gamma_{\beta}$ with respect to the variable $\operatorname{Im} w \in \mathbb{R}$ are denoted in the usual manner with $\Gamma_{\beta}$ instead of $\mathbb{R}$ ). Together with the inverse $M_{\gamma}^{-1}$ we can form Mellin pseudo-differential operators

$$
\mathrm{op}_{M}^{\gamma}(f) u(r):=\iint_{0}^{\infty}\left(\frac{r}{r^{\prime}}\right)^{-\left(\frac{1}{2}-\gamma+i \rho\right)} f\left(r, r^{\prime}, w\right) u\left(r^{\prime}\right) \frac{d r^{\prime}}{r^{\prime}} d \rho,
$$


$\Varangle \rho:=(2 \pi)^{-1} d \rho$, for any amplitude function $f\left(r, r^{\prime}, w\right) \in C^{\infty}\left(\mathbb{R}_{+} \times \mathbb{R}_{+}\right.$, $S^{\mu}\left(\Gamma_{\frac{1}{2}-\gamma}\right)$ ) (with $S^{\mu}\left(\Gamma_{\beta}\right)$ denoting Hörmander's space of symbols of order $\mu$ with constant coefficients and covariable $\operatorname{Im} w \in \Gamma_{\beta}$ ), cf. also Definition 1.5 below.

In this exposition by a cut-off function $\omega(r)$ on the half axis we understand any $\omega \in C_{0}^{\infty}\left(\overline{\mathbb{R}}_{+}\right)$which is equal to 1 near $r=0$.

Definition 1.1 For $s, \gamma \in \mathbb{R}$ we define

(i) $\mathcal{H}^{s, \gamma}\left(\mathbb{R}_{+}\right)$to be the completion of $C_{0}^{\infty}\left(\mathbb{R}_{+}\right)$with respect to the norm

$$
\left\{\frac{1}{2 \pi i} \int_{\Gamma_{\frac{1}{2}-\gamma}}\left(1+|w|^{2}\right)^{s}\left|M_{\gamma} u(w)\right|^{2} d w\right\}^{\frac{1}{2}}
$$

(ii) $\mathcal{K}^{s, \gamma}\left(\mathbb{R}_{+}\right):=\left\{\omega u+(1-\omega) v: u \in \mathcal{H}^{s, \gamma}\left(\mathbb{R}_{+}\right), v \in H^{s}\left(\mathbb{R}_{+}\right)\right\}$

for any cut-off function $\omega$.

More generally, we form the space $\mathcal{H}^{s, \gamma}\left(\mathbb{R}_{+} \times \mathbb{R}\right)$ as the completion of $C_{0}^{\infty}\left(\mathbb{R}_{+} \times \mathbb{R}\right)$ with respect to the norm

$$
\left\{\frac{1}{2 \pi i} \int_{\mathbb{R}} \int_{\Gamma_{1-\gamma}}\left(1+|w|^{2}+|\vartheta|^{2}\right)^{s}\left|M_{\gamma} F u(w, \vartheta)\right|^{2} d w d \vartheta\right\}^{\frac{1}{2}}
$$

with the Fourier transform $F=F_{\phi \rightarrow \vartheta}$ on $\mathbb{R}$ and set

$$
\mathcal{H}^{s, \gamma}\left(I_{\alpha}^{\wedge}\right):=\left.\mathcal{H}^{s, \gamma}\left(\mathbb{R}_{+} \times \mathbb{R}\right)\right|_{\mathbb{R}_{+} \times(0, \alpha)} .
$$

Moreover, let

$$
\mathcal{K}^{s, \gamma}\left(I_{\alpha}^{\wedge}\right):=\left\{\omega u+(1-\omega) v: u \in \mathcal{H}^{s, \gamma}\left(I_{\alpha}^{\wedge}\right),\left.v \in H^{s}\left(\mathbb{R}^{2}\right)\right|_{\operatorname{int} K_{\alpha}}\right\}
$$

for any cut-off function $\omega(r)$ on the half-axis, and $\alpha<2 \pi$. In this definition we tacitly identify $I_{\alpha}^{\wedge}$ with the angle $K_{\alpha} \subset \mathbb{R}^{2}$ and interpret $1-\omega$ as $1-$ $\omega(|x|), x \in \mathbb{R}^{2}$. The case $\alpha=2 \pi$ needs a separate definition, since $K_{2 \pi}$ consists of the plane with a slit along the positive $x_{1}$-half-axis. But this case can be covered as well by taking sums of corresponding elements supported by $[\varepsilon, \pi]$ and $[-\varepsilon,-\pi]$ for some $0<\varepsilon<\pi$.

The spaces $\mathcal{H}^{s, \gamma}(\cdot)$ and $\mathcal{K}^{s, \gamma}(\cdot)$ can be endowed with Hilbert space scalar products and corresponding norms.

In the sequel we identify $I_{ \pm}$with $\mathbb{R}_{ \pm}$. Observe that the operators $\mathrm{r}_{ \pm}$: $C_{0}^{\infty}\left(I_{\alpha}^{\wedge}\right) \rightarrow C_{0}^{\infty}\left(\mathbb{R}_{ \pm}\right)$extend to continuous operators

$$
\mathrm{r}_{ \pm}: \mathcal{K}^{s, \gamma}\left(I_{\alpha}^{\wedge}\right) \rightarrow \mathcal{K}^{s-\frac{1}{2}, \gamma-\frac{1}{2}}\left(\mathbb{R}_{ \pm}\right), \quad \mathcal{H}^{s, \gamma}\left(I_{\alpha}^{\wedge}\right) \rightarrow \mathcal{H}^{s-\frac{1}{2}, \gamma-\frac{1}{2}}\left(\mathbb{R}_{ \pm}\right)
$$

for every $s>\frac{1}{2}, \gamma \in \mathbb{R}$. With the operator $\mathcal{A}={ }^{\mathrm{t}}\left(\begin{array}{lll}A & T_{-} & T_{+}\end{array}\right)$we associate the so-called principal edge symbol

$$
\sigma_{\wedge}(\mathcal{A})(\eta):=\left(\sigma_{\wedge}(A)(\eta) \quad \sigma_{\wedge}\left(T_{-}\right)(\eta) \quad \sigma_{\wedge}\left(T_{+}\right)(\eta)\right)
$$


$\eta \neq 0$, where

$$
\begin{aligned}
\sigma_{\wedge}(A)(\eta) & :=r^{-2}\left(\left(r \partial_{r}\right)^{2}+\partial_{\phi}^{2}-r^{2}|\eta|^{2}\right) \\
\sigma_{\wedge}\left(T_{ \pm}\right)(\eta) & :=\mathrm{r}_{ \pm} r^{-\mu_{ \pm}} \sum_{j+|\beta| \leq \mu_{ \pm}} b_{ \pm, j \beta}\left(-r \partial_{r}\right)^{j}(r \eta)^{\beta}
\end{aligned}
$$

It represents a family of continuous operators

$$
\begin{aligned}
\sigma_{\wedge}(\mathcal{A})(\eta) \quad: \quad \mathcal{K}^{s, \gamma}\left(I_{\alpha}^{\wedge}\right) \rightarrow \mathcal{K}^{s-2, \gamma-2}\left(I_{\alpha}^{\wedge}\right) \\
\oplus \mathcal{K}^{s-\mu_{-}-\frac{1}{2}, \gamma-\mu_{-}-\frac{1}{2}}\left(\mathbb{R}_{-}\right) \oplus \mathcal{K}^{s-\mu_{+}-\frac{1}{2}, \gamma-\mu_{+}-\frac{1}{2}}\left(\mathbb{R}_{+}\right)
\end{aligned}
$$

for arbitrary $s>\max \left\{\mu_{-}+\frac{1}{2}, \mu_{+}+\frac{1}{2}\right\}$. If we endow the $\mathcal{K}^{s, \gamma}$-spaces with the group actions

$$
\begin{aligned}
\kappa_{\lambda}^{(1)}: u(r, \phi) & \rightarrow \lambda u(\lambda r, \phi), \quad u \in \mathcal{K}^{s, \gamma}\left(I_{\alpha}^{\wedge}\right), \\
\kappa_{\lambda}^{(0)}: v(r) & \rightarrow \lambda^{\frac{1}{2}} v(\lambda r), \quad v \in \mathcal{K}^{s, \gamma}\left(\mathbb{R}_{ \pm}\right),
\end{aligned}
$$

$\lambda \in \mathbb{R}_{+}$(cf. also Section 1.3 below), we obtain the (so-called twisted) homogeneities

$$
\begin{aligned}
\sigma_{\wedge}(A)(\lambda \eta) & =\lambda^{2} \kappa_{\lambda}^{(1)} \sigma_{\wedge}(A)(\eta)\left(\kappa_{\lambda}^{(1)}\right)^{-1} \\
\sigma_{\wedge}\left(T_{ \pm}\right)(\lambda \eta) & =\lambda^{\mu_{ \pm}+\frac{1}{2}} \kappa_{\lambda}^{(0)} \sigma_{\wedge}\left(T_{ \pm}\right)(\eta)\left(\kappa_{\lambda}^{(1)}\right)^{-1}
\end{aligned}
$$

for all $\lambda \in \mathbb{R}_{+}$. For every fixed $\eta \neq 0$ the operators $\sigma_{\wedge}(\mathcal{A})(\eta)$ are boundary value problems on the infinite cone $K_{\alpha}$ which belong to the cone algebra. As such they have the principal symbols of that calculus, especially, the so-called conormal symbol, defined as

$$
\sigma_{\mathrm{c}} \sigma_{\wedge}(\mathcal{A})(w):={ }^{\mathrm{t}}\left(\sigma_{\mathrm{c}} \sigma_{\wedge}(A)(w) \quad \sigma_{\mathrm{c}} \sigma_{\wedge}\left(T_{-}\right)(w) \quad \sigma_{\mathrm{c}} \sigma_{\wedge}\left(T_{+}\right)(w)\right)
$$

where

$$
\begin{gathered}
\sigma_{\mathrm{c}} \sigma_{\wedge}(A)(w):=w^{2}+\partial_{\phi}^{2}: H^{s}\left(\operatorname{int} I_{\alpha}\right) \rightarrow H^{s-2}\left(\operatorname{int} I_{\alpha}\right), \\
\sigma_{\mathrm{c}} \sigma_{\wedge}\left(T_{ \pm}\right)(w):=\mathrm{r}_{ \pm} \sum_{j=0}^{\mu_{ \pm}} b_{ \pm, j 0} w^{j}: H^{s}\left(\operatorname{int} I_{\alpha}\right) \rightarrow \mathbb{C}
\end{gathered}
$$

$w \in \mathbb{C}$, here with $\mathbf{r}_{ \pm}$in the meaning of the restriction to the end points $\left\{\alpha_{ \pm}\right\}$ of the interval $I_{\alpha}$.

In Section 2 below we will characterise the Fredholm property and the index of $\sigma_{\wedge}(\mathcal{A})(\eta)$ under standard ellipticity conditions for $T_{ \pm}$, together with the non-bijectivity points of $\sigma_{\mathrm{c}} \sigma_{\wedge}(\mathcal{A})(w)$ in the complex plane.

Remark 1.2 For every $s \in \mathbb{R}$ there is a family of isomorphisms

$$
L(\beta, \delta ; \alpha, \gamma): \mathcal{K}^{s, \gamma}\left(I_{\alpha}^{\wedge}\right) \rightarrow \mathcal{K}^{s, \delta}\left(I_{\beta}^{\wedge}\right)
$$


for every fixed $\delta \in \mathbb{R}$ and $\beta \in(0,2 \pi]$ which can be constructed by a simple dilation of the opening angle $\alpha$ and multiplying functions by a factor $\omega(r) r^{\delta-\gamma}+(1-\omega(r))$. This is particularly simple for $0<\alpha<2 \pi, 0<\beta<2 \pi$, what concerns the contribution of $\left.H^{s}\left(\mathbb{R}^{2}\right)\right|_{\operatorname{int} K_{\alpha}}$, see the formula (8), but a simple modification allows us to assume also the opening angles to be

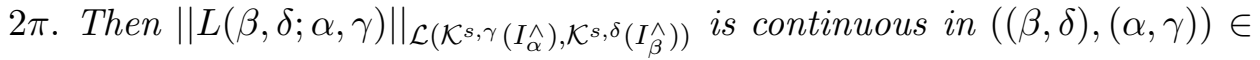
$(0,2 \pi] \times \mathbb{R} \times(0,2 \pi] \times \mathbb{R}$.

\subsection{Weighted edge spaces and symbols with twisted homo- geneity}

We now establish so-called weighted edge spaces in which our boundary value problems are expected to have a parametrix.

A Hilbert space $E$ is said to be equipped with a group action $\kappa=$ $\left\{\kappa_{\lambda}\right\}_{\lambda \in \mathbb{R}_{+}}$if $\kappa_{\lambda}: E \rightarrow E, \lambda \in \mathbb{R}_{+}$, is a group of isomorphisms, $\kappa_{\lambda} \kappa_{\lambda^{\prime}}=$ $\kappa_{\lambda \lambda^{\prime}}, \lambda, \lambda^{\prime} \in \mathbb{R}_{+}$, and if $\lambda \rightarrow \kappa_{\lambda} e$ defines a continuous map $\mathbb{R}_{+} \rightarrow E$ for every $e \in E$.

We then denote by $\mathcal{W}^{s}\left(\mathbb{R}^{q}, E\right), s \in \mathbb{R}$, the completion of $\mathcal{S}\left(\mathbb{R}^{q}, E\right)$ (the space of Schwartz functions with values in $E$ ) with respect to the norm $\left\|\langle\eta\rangle^{s} \kappa_{\langle\eta\rangle}^{-1} \hat{u}(\eta)\right\|_{L^{2}\left(\mathbb{R}^{q}, E\right)}$, where $\hat{u}=F_{y \rightarrow \eta} u$ is the Fourier transform in $\mathbb{R}^{q}$ (applied to vector-valued functions), and $\kappa_{\langle\eta\rangle}^{-1}$ acts on the values of $\hat{u}(\eta)$ (as usual, $\left.\langle\eta\rangle:=\left(1+|\eta|^{2}\right)^{\frac{1}{2}}\right)$. If necessary, we also write $\mathcal{W}^{s}\left(\mathbb{R}^{q}, E\right)_{\kappa}$ if we want to indicate the dependence of the space on the choice of $\kappa$.

Example 1.3 Let $g \in \mathbb{R}$ and set

$$
\mathcal{K}^{s, \gamma ; g}\left(I_{\alpha}^{\wedge}\right):=\langle r\rangle^{-g} \mathcal{K}^{s, \gamma}\left(I_{\alpha}^{\wedge}\right)
$$

$s, \gamma, g \in \mathbb{R}$, endowed with the group action

$$
\kappa_{\lambda}^{(1), g}: u(r, \phi) \rightarrow \lambda^{1+g} u(\lambda r, \phi)
$$

Then we can form the weighted edge spaces

$$
\mathcal{W}^{s}\left(\mathbb{R}^{q}, \mathcal{K}^{s, \gamma ; g}\left(I_{\alpha}^{\wedge}\right)\right)_{\kappa^{(1), g}} .
$$

In a similar manner we have $\mathcal{K}^{s, \gamma ; g}\left(\mathbb{R}_{ \pm}\right):=\langle r\rangle^{-g} \mathcal{K}^{s, \gamma}\left(\mathbb{R}_{ \pm}\right), \kappa_{\lambda}^{(0), g}: u(r) \rightarrow$ $\lambda^{\frac{1}{2}+g} u(\lambda r)$, with the spaces $\mathcal{W}^{s}\left(\mathbb{R}^{q}, \mathcal{K}^{s, \gamma ; g}\left(\mathbb{R}_{ \pm}\right)\right)_{\kappa^{(0), g}}$.

Remark 1.4 The general definition of the spaces $\mathcal{W}^{s}\left(\mathbb{R}^{q}, E\right)$ was introduced in [14], based on the observations of [13] for the case $E=\mathcal{K}^{s, \gamma}\left(X^{\wedge}\right)$, and then systematically employed in the pseudo-differential edge calculus. Here $X$ is an arbitrary compact $C^{\infty}$ manifold (with or without boundary), and $\mathcal{K}^{s, \gamma}\left(X^{\wedge}\right)$ are natural analogues of the former $\mathcal{K}^{s, \gamma}$-spaces on the open infinite stretched cone $X^{\wedge}=\mathbb{R}_{+} \times X$; the group action $\kappa=\left\{\kappa_{\lambda}\right\}_{\lambda \in \mathbb{R}_{+}}$on 
$\mathcal{K}^{s, \gamma}\left(X^{\wedge}\right)$ is defined by $\kappa_{\lambda}: u(r, x) \rightarrow \lambda^{\frac{n+1}{2}} u(\lambda r, x), \lambda \in \mathbb{R}_{+}, n=\operatorname{dim} X, c f$. [15], [3], [9]. Certain applications of weighted edge spaces to hyperbolic problems, see Airapetyan and Witt [1], suggested to modify the spaces $\mathcal{K}^{s, \gamma}\left(X^{\wedge}\right)$ to $\mathcal{K}^{s, \gamma ; g}\left(X^{\wedge}\right):=\langle r\rangle^{-g} \mathcal{K}^{s, \gamma}\left(X^{\wedge}\right)$ for certain $g \in \mathbb{R}$, with a corresponding adapted group action, namely, $\kappa_{\lambda}^{g}: u(r, x) \rightarrow \lambda^{g+\frac{n+1}{2}} u(\lambda r, x)$. Motivated by the question of invariance with respect to the transition maps of a maximal system of singular wedge charts Tarkhanov [18] studied the case $g=s-\gamma$ and verified the property

$$
H_{\text {comp }}^{s}\left(X^{\wedge} \times \mathbb{R}^{q}\right) \subset \mathcal{W}^{s}\left(\mathbb{R}^{q}, \mathcal{K}^{s, \gamma ; g}\left(X^{\wedge}\right)\right)_{\kappa^{g}} \subset H_{\text {loc }}^{s}\left(X^{\wedge} \times \mathbb{R}^{q}\right) .
$$

For the case $g=0$ the relation (15) was checked before in [15]. In [7, Section 7.1.2] the case of arbitrary $g \in \mathbb{R}$ is studied, and the property (15) is shown. On the other hand the spaces

$$
\mathcal{W}^{s}\left(\mathbb{R}^{q}, \mathcal{K}^{s, \gamma ; g}\left(X^{\wedge}\right)\right)_{\kappa^{g}}
$$

are mutually non-equivalent for different $g$, although outside any neighbourhood of the edge $\mathbb{R}^{q}$ they coincide in the sense of (15). Concerning other nice properties of the spaces (16) for $g=s-\mu$, see [18] and also [16].

Definition 1.5 Let $E$ and $\tilde{E}$ be Hilbert spaces with group action $\kappa$ and $\tilde{\kappa}$, respectively, and let $\mu \in \mathbb{R}, U \subseteq \mathbb{R}^{p}$ open.

(i) The space $S^{\mu}\left(U \times \mathbb{R}^{q} ; E, \tilde{E}\right)$ of symbols of order $\mu$ is defined to be the set of all smooth functions $a: U_{y} \times \mathbb{R}_{\eta}^{q} \rightarrow \mathcal{L}(E, \tilde{E})$, such that

$$
\sup _{y \in K, \eta \in \mathbb{R}^{q}}\langle\eta\rangle^{-\mu+|\beta|}\left\|\tilde{\kappa}_{\langle\eta\rangle}^{-1}\left\{D_{y}^{\alpha} D_{\eta}^{\beta} a(y, \eta)\right\} \kappa_{\langle\eta\rangle}\right\|_{\mathcal{L}(E, \tilde{E})}
$$

is finite for every $K \subset \subset U$, and multi-indices $\alpha \in \mathbb{N}^{p}, \beta \in \mathbb{N}^{q}$.

(ii) We define $S^{(\mu)}\left(U \times\left(\mathbb{R}^{q} \backslash\{0\}\right) ; E, \tilde{E}\right)$ as the set of all smooth $a_{(\mu)}$ : $U \times\left(\mathbb{R}^{q} \backslash\{0\}\right) \rightarrow \mathcal{L}(E, \tilde{E})$ such that

$$
a_{(\mu)}(y, \lambda \eta)=\lambda^{\mu} \tilde{\kappa}_{\lambda} a_{(\mu)}(y, \eta) \kappa_{\lambda}^{-1}
$$

for all $\lambda \in \mathbb{R}_{+},(y, \eta) \in U \times\left(\mathbb{R}^{q} \backslash\{0\}\right)$.

(iii) $S_{\mathrm{cl}}^{\mu}\left(U \times \mathbb{R}^{q} ; E, \tilde{E}\right)$ is defined to be the subspace of all $a(y, \eta)$ as in (i) such that for any excision function $\chi(\eta)$ (i.e., $\chi \in C^{\infty}\left(\mathbb{R}^{q}\right), \chi(\eta)=0$ for $|\eta|<c_{0}, \chi(\eta)=1$ for $|\eta|>c_{1}$ for some constants $\left.0<c_{0}<c_{1}\right)$ we have $a(y, \eta)-\chi(\eta) \sum_{j=0}^{N} a_{(\mu-j)}(y, \eta) \in S^{\mu-(N+1)}\left(U \times \mathbb{R}^{q} ; E, \tilde{E}\right)$ for every $N \in \mathbb{N}$.

If we want to indicate the dependence of the symbol spaces on $\kappa, \tilde{\kappa}$ we also write

$$
S_{(\mathrm{cl})}^{\mu}\left(U \times \mathbb{R}^{q} ; E, \tilde{E}\right)_{\kappa, \tilde{\kappa}}
$$


rather than $S_{(\mathrm{cl})}^{\mu}\left(U \times \mathbb{R}^{q} ; E, \tilde{E}\right)$ (subscript '(cl)' is used when a consideration is valid both in the classical and non-classical case).

By $S_{(\mathrm{cl})}^{\mu}\left(\mathbb{R}^{q} ; E, \tilde{E}\right)$ we denote the subspace of symbols with constant coefficients (i.e., independent of $y \in U$ ).

Example $1.6 \quad$ (i) For the operator (1) we have

$$
\sigma_{\wedge}(A)(\eta) \in S_{\mathrm{cl}}^{2}\left(\mathbb{R}^{q} ; \mathcal{K}^{s, \gamma ; g}\left(I_{\alpha}^{\wedge}\right), \mathcal{K}^{s-2, \gamma-2 ; g}\left(I_{\alpha}^{\wedge}\right)\right)
$$

for every $s, \gamma, g \in \mathbb{R}$.

(ii) For the operators (2) (and, for simplicity, constant $b_{ \pm, j \beta}$ ) we have

$$
\sigma_{\wedge}\left(T_{ \pm}\right)(\eta) \in S_{\mathrm{cl}}^{\mu_{ \pm}+\frac{1}{2}}\left(\mathbb{R}^{q} ; \mathcal{K}^{s, \gamma ; g}\left(I_{\alpha}^{\wedge}\right), \mathcal{K}^{s-\mu_{ \pm}-\frac{1}{2}, \gamma-\mu_{ \pm}-\frac{1}{2} ; g}\left(\mathbb{R}_{ \pm}\right)\right)
$$

for every $s>\max \left\{\mu_{-}+\frac{1}{2}, \mu_{+}+\frac{1}{2}\right\}, \gamma, g \in \mathbb{R}$.

Remark 1.7 Let $E, \kappa$ and $\tilde{E}, \tilde{\kappa}$ be as in Definition $1.5, a(\eta) \in S^{\mu}\left(\mathbb{R}^{q}\right.$; $E, \tilde{E})_{\kappa, \tilde{\kappa}}$. Then $\operatorname{Op}(a)$ defined by

$$
\mathrm{Op}(a) u(y)=\iint e^{i\left(y-y^{\prime}\right) \eta} a(\eta) u\left(y^{\prime}\right) d y^{\prime} d \eta,
$$

$d \eta:=(2 \pi)^{-q} d \eta$, induces a continuous operator

$$
\mathrm{Op}(a): \mathcal{W}^{s}\left(\mathbb{R}^{q}, E\right)_{\kappa} \rightarrow \mathcal{W}^{s-\mu}\left(\mathbb{R}^{q}, \tilde{E}\right)_{\tilde{\kappa}}
$$

for every $s \in \mathbb{R}$. There are continuity results of a similar kind for much more general symbols a, especially, with variable coefficients, see [15] or [17]. However, for the main purposes here it suffices the case with constant coefficients.

Proposition 1.8 The operators in the boundary value problem (6) induce a continuous operator

$$
\begin{gathered}
\mathcal{W}^{s-2}\left(\mathbb{R}^{q}, \mathcal{K}^{s-2, \gamma-2 ; g}\left(I_{\alpha}^{\wedge}\right)\right) \\
\mathcal{A}: \mathcal{W}^{s}\left(\mathbb{R}^{q}, \mathcal{K}^{s, \gamma ; g}\left(I_{\alpha}^{\wedge}\right)\right) \rightarrow \mathcal{W}^{s-\mu_{-}-\frac{1}{2}}\left(\mathbb{R}^{q}, \mathcal{K}^{\left.s-\mu_{--\frac{1}{2}, \gamma-\mu_{-}-\frac{1}{2} ; g}\left(\mathbb{R}_{-}\right)\right)}\right. \\
\mathcal{W}^{s-\mu_{+}-\frac{1}{2}}\left(\mathbb{R}^{q}, \mathcal{K}^{s-\mu_{+}-\frac{1}{2}, \gamma-\mu_{+}-\frac{1}{2} ; g}\left(\mathbb{R}_{+}\right)\right)
\end{gathered}
$$

for every $s>\max \left\{\mu_{-}+\frac{1}{2}, \mu_{+}+\frac{1}{2}\right\}, \gamma, g \in \mathbb{R}$. (The weighted edge spaces refer to the group actions $\kappa^{(1), g}$ and $\kappa^{(0), g}$, respectively.)

Proof. The result immediately follows from Remark 1.7 together with Example 1.6. 


\section{Fredholm property and index of edge symbols}

\subsection{Ellipticity on the infinite cone}

As noted before the operators (10) belong to the cone algebra of boundary value problems on the infinite stretched cone $I_{\alpha}^{\wedge}$ with the boundary components $\mathbb{R}_{ \pm}$, for every fixed $\eta \neq 0$. Concerning the boundary conditions we assume (3), (4), or (5); they satisfy the Shapiro-Lopatinskij condition. For the orders $\mu_{ \pm}$we have

$$
\mu_{ \pm}=0 \text { for }(3), \quad \mu_{ \pm}=1 \text { for (4), }
$$

and

$$
\mu_{-}=0, \quad \mu_{+}=1 \text { for }(5) .
$$

The general formulations are valid for all these cases.

On the infinite stretched cone $I_{\alpha}^{\wedge}$ we have the principal symbolic hierarchy of operators of the cone calculus, where $r \rightarrow \infty$ is treated as a conical exit of the configuration to $\infty$, see, for instance, [9, Section 3.3], namely,

$$
\sigma\left(\sigma_{\wedge}(\mathcal{A})\right):=\left(\sigma_{\psi} \sigma_{\wedge}(\mathcal{A}), \sigma_{\partial} \sigma_{\wedge}(\mathcal{A}), \sigma_{\mathrm{c}} \sigma_{\wedge}(\mathcal{A}), \sigma_{\mathrm{E}} \sigma_{\wedge}(\mathcal{A}), \sigma_{\mathrm{E}^{\prime}} \sigma_{\wedge}(\mathcal{A})\right)
$$

$\sigma_{\wedge}(\mathcal{A})=\sigma_{\wedge}(\mathcal{A})(\eta)={ }^{\mathrm{t}}\left(\sigma_{\wedge}(A)(\eta) \quad \sigma_{\wedge}\left(T_{-}\right)(\eta) \quad \sigma_{\wedge}\left(T_{+}\right)(\eta)\right)$ for fixed $\eta \neq 0$. The meaning of the components is as follows:

(i) the interior symbol $\sigma_{\psi} \sigma_{\wedge}(\mathcal{A})(r, \rho, \vartheta):=r^{-2}\left((-i r \rho)^{2}-\vartheta^{2}\right)$;

(ii) the boundary symbol

$$
\sigma_{\partial, \pm} \sigma_{\wedge}(A)(r, \rho):=r^{-2}\left((-i r \rho)^{2}+\partial_{\phi}^{2}\right): H^{s}\left(\mathbb{R}_{+}\right) \rightarrow H^{s-2}\left(\mathbb{R}_{+}\right)
$$

(referring to $\left.(r, \rho) \in T^{*} I_{ \pm} \backslash 0\right)$,

$$
\sigma_{\partial} \sigma_{\wedge}\left(T_{ \pm}\right)(r, \rho)=\mathrm{r}_{ \pm} r^{-\mu_{ \pm}} \sum_{j=0}^{\mu_{ \pm}} b_{ \pm, j 0}(-i r \rho)^{j}: H^{s}\left(\mathbb{R}_{+}\right) \rightarrow \mathbb{C}
$$

with $b_{ \pm, j 0}$ being interpreted as differential operators with respect to $\phi \in I_{\alpha} ;$

(iii) the conormal symbol is given by the formulas (11), (12), (13) and defines continuous operators

$$
\sigma_{\mathrm{c}} \sigma_{\wedge}(\mathcal{A})(w): H^{s}\left(\operatorname{int} I_{\alpha}\right) \rightarrow H^{s-2}\left(\operatorname{int} I_{\alpha}\right) \oplus \mathbb{C}^{2} ;
$$

(iv) the interior exit symbol, expressed in Euclidean coordinates $x \in K_{\alpha}$ far from $x=0$

$$
\sigma_{\mathrm{E}} \sigma_{\wedge}(\mathcal{A}):=\sigma_{\mathrm{E}} \sigma_{\wedge}(A)
$$

with the components

$$
\sigma_{E_{1}} \sigma_{\wedge}(\mathcal{A}):=-|\xi|^{2}-|\eta|^{2}, \quad \sigma_{E_{2}} \sigma_{\wedge}(\mathcal{A}):=-|\xi|^{2} ;
$$


(v) the boundary exit symbol in Euclidean coordinates $x \in K_{\alpha}$ far from $x=0$

$$
\sigma_{E^{\prime}, \pm} \sigma_{\wedge}(\mathcal{A}):={ }^{\mathrm{t}}\left(\sigma_{E^{\prime}} \sigma_{\wedge}(A) \quad \sigma_{E^{\prime}} \sigma_{\wedge}\left(T_{ \pm}\right)\right)
$$

which we express for the boundary component $I_{-}$that may be identified with the positive $x_{1}$-half-axis (for the component over $I_{+}$the symbols can be considered by a rotation such that it also coincides with $\mathbb{R}_{x_{1},+}$ and its behaviour is then analogous). We then have

$$
\begin{aligned}
& \sigma_{\mathrm{E}_{1}^{\prime}} \sigma_{\wedge}(A)=-\left|\xi_{1}\right|^{2}+\frac{\partial^{2}}{\partial x_{2}^{2}}-|\eta|^{2}: H^{s}\left(\mathbb{R}_{+}\right) \rightarrow H^{s-2}\left(\mathbb{R}_{+}\right), \\
& \sigma_{\mathrm{E}_{2}^{\prime}} \sigma_{\wedge}(A)=-\left|\xi_{1}\right|^{2}+\frac{\partial^{2}}{\partial x_{2}^{2}}: H^{s}\left(\mathbb{R}_{+}\right) \rightarrow H^{s-2}\left(\mathbb{R}_{+}\right) .
\end{aligned}
$$

For $\sigma_{\wedge}(T)$ we consider, for instance, the case (5) where

$$
\sigma_{\mathrm{E}_{j}^{\prime}} \sigma_{\wedge}\left(T_{-}\right)=\mathrm{r}_{0}
$$

and (after the above mentioned rotation)

$$
\sigma_{\mathrm{E}_{j}^{\prime}} \sigma_{\wedge}\left(T_{+}\right)=\mathrm{r}_{0} \frac{\partial}{\partial x_{2}}
$$

with $\mathrm{r}_{0}$ denoting the operator of restriction to $x_{2}=0$,

$$
\sigma_{\mathrm{E}_{j}^{\prime}} \sigma_{\wedge}\left(T_{ \pm}\right): H^{s}\left(\mathbb{R}_{+}\right) \rightarrow \mathbb{C}
$$

$j=1,2$.

Theorem 2.1 The operator (10) for any fixed $\eta \neq 0$ is Fredholm for $s>\frac{3}{2}$ and for those $\gamma \in \mathbb{R}$ where

$$
\sigma_{\mathrm{c}} \sigma_{\wedge}(\mathcal{A})(w): H^{s}\left(\operatorname{int} I_{\alpha}\right) \rightarrow H^{s-2}\left(\operatorname{int} I_{\alpha}\right) \oplus \mathbb{C}^{2}
$$

is a family of isomorphisms for all $w \in \Gamma_{1-\gamma}$.

Proof. Let us consider the case of boundary conditions (5) (the cases (3), (4) can be treated in an analogous manner). From the general calculus of boundary value problems on an infinite cone (see [9, Section 2.2 and 3.3]) it is known that the operator (10) is Fredholm if and only if all components of (19) are elliptic. For $\sigma_{\mathrm{c}} \sigma_{\wedge}(\mathcal{A})$ that is exactly the condition formulated in the theorem. The ellipticity of the other components is to be understood as follows.

(i) $\sigma_{\psi} \sigma_{\wedge}(\mathcal{A})(r, \rho, \vartheta) \neq 0$ for $(\rho, \vartheta) \neq 0$ and for all $r \in \mathbb{R}_{+}, \tilde{\sigma}_{\psi} \sigma_{\wedge}(\mathcal{A})(r, \rho, \vartheta)$ $:=r^{2} \sigma_{\psi} \sigma_{\wedge}(\mathcal{A})\left(r, r^{-1} \rho, \vartheta\right) \neq 0$ for $(\rho, \vartheta) \neq 0$ and for all $r \in \mathbb{R}_{+}$, up to $r=0$. 
(ii) $\sigma_{\partial, \pm} \sigma_{\wedge}(\mathcal{A})(r, \rho): H^{s}\left(\mathbb{R}_{+}\right) \rightarrow H^{s-2}\left(\mathbb{R}_{+}\right) \oplus \mathbb{C}$ is an isomorphism for all $(r, \rho) \in T^{*} I_{ \pm} \backslash 0$

$$
\begin{aligned}
\tilde{\sigma}_{\partial, \pm} \sigma_{\wedge}(\mathcal{A})(r, \rho):= & { }^{\mathrm{t}}\left(r^{2} \sigma_{\partial} \sigma_{\wedge}(A)\left(r, r^{-1} \rho\right) \quad r^{\mu_{ \pm}} \sigma_{\partial} \sigma_{\wedge}\left(T_{ \pm}\right)\left(r, r^{-1} \rho\right)\right) \\
& : \quad H^{s}\left(\mathbb{R}_{+}\right) \rightarrow H^{s-2}\left(\mathbb{R}_{+}\right) \oplus \mathbb{C}
\end{aligned}
$$

is an isomorphism for all $\rho \neq 0$, up to $r=0$, in both cases for $s>\frac{3}{2}$.

(iv)

$$
\begin{aligned}
& \sigma_{E_{1}} \sigma_{\wedge}(\mathcal{A})(\eta)(\xi) \neq 0 \text { for all } \xi \in \mathbb{R}^{2}, \\
& \sigma_{E_{2}} \sigma_{\wedge}(\mathcal{A})(\xi) \neq 0 \text { for all } \xi \in \mathbb{R}^{2} \backslash\{0\}
\end{aligned}
$$

(v) For the minus component:

$$
\begin{aligned}
\sigma_{E_{1}^{\prime},-} \sigma_{\wedge}(\mathcal{A})(\eta)\left(\xi_{1}\right) & ={ }^{\mathrm{t}}\left(-\left|\xi_{1}\right|^{2}+\partial_{x_{2}}^{2}-|\eta|^{2} \quad \mathrm{r}_{0}\right) \\
& : \quad H^{s}\left(\mathbb{R}_{+}\right) \rightarrow H^{s-2}\left(\mathbb{R}_{+}\right) \oplus \mathbb{C}
\end{aligned}
$$

is an isomorphism for all $\xi_{1} \in \mathbb{R}$,

$$
\begin{aligned}
\sigma_{E_{2}^{\prime},-} \sigma_{\wedge}(\mathcal{A})\left(\xi_{1}\right)= & { }^{\mathrm{t}}\left(-\left|\xi_{1}\right|^{2}+\partial_{x_{2}}^{2} \quad \mathrm{r}_{0}\right) \\
& : \quad H^{s}\left(\mathbb{R}_{+}\right) \rightarrow H^{s-2}\left(\mathbb{R}_{+}\right) \oplus \mathbb{C}
\end{aligned}
$$

is an isomorphism for all $\xi_{1} \in \mathbb{R} \backslash\{0\}$, in both cases for $s>\frac{3}{2}$.

The condition for the +-component is analogous. It remains to observe that (i), (ii), (iv), and (v) are satisfied for our problem.

\subsection{The index of edge symbols}

We now compute the index of $(10), \eta \neq 0$, for the case of the boundary conditions (3), (4), and (5). To this end we first establish the weights where the corresponding conormal symbols (20) are bijective.

In order to distinguish the Dirichlet, Neumann, and Zaremba case (3), (4), and (5), we now write for the corresponding operators $\mathcal{A}_{1}, \mathcal{A}_{2}$, and $\mathcal{A}_{3}$, respectively.

Let us set

$$
\mathcal{C}_{i}:=\sigma_{\wedge}\left(\mathcal{A}_{i}\right)(0), i=1,2,3,
$$

which can be interpreted as continuous operators

$$
\mathcal{C}_{i}: \mathcal{H}^{s, \gamma}\left(I_{\alpha}^{\wedge}\right) \rightarrow \mathcal{H}_{i}^{s-2, \gamma-2}\left(I_{\alpha}^{\wedge}\right),
$$

$i=1,2,3$, where

$$
\begin{aligned}
& \mathcal{H}_{1}^{s-2, \gamma-2}\left(I_{\alpha}^{\wedge}\right):=\mathcal{H}^{s-2, \gamma-2}\left(I_{\alpha}^{\wedge}\right) \oplus \mathcal{H}^{s-\frac{1}{2}, \gamma-\frac{1}{2}}\left(\mathbb{R}_{-}\right) \oplus \mathcal{H}^{s-\frac{1}{2}, \gamma-\frac{1}{2}}\left(\mathbb{R}_{+}\right), \\
& \mathcal{H}_{2}^{s-2, \gamma-2}\left(I_{\alpha}^{\wedge}\right):=\mathcal{H}^{s-2, \gamma-2}\left(I_{\alpha}^{\wedge}\right) \oplus \mathcal{H}^{s-\frac{3}{2}, \gamma-\frac{3}{2}}\left(\mathbb{R}_{-}\right) \oplus \mathcal{H}^{s-\frac{3}{2}, \gamma-\frac{3}{2}}\left(\mathbb{R}_{+}\right), \\
& \mathcal{H}_{3}^{s-2, \gamma-2}\left(I_{\alpha}^{\wedge}\right):=\mathcal{H}^{s-2, \gamma-2}\left(I_{\alpha}^{\wedge}\right) \oplus \mathcal{H}^{s-\frac{1}{2}, \gamma-\frac{1}{2}}\left(\mathbb{R}_{-}\right) \oplus \mathcal{H}^{s-\frac{3}{2}, \gamma-\frac{3}{2}}\left(\mathbb{R}_{+}\right) .
\end{aligned}
$$


Theorem 2.2 For every $s>\frac{3}{2}$ the operators (22) are isomorphisms for

(i) the case $i=1$ for all $\gamma \neq \frac{k \pi}{\alpha}+1, k \in \mathbb{Z} \backslash\{0\}$;

(ii) the case $i=2$ for all $\gamma \neq \frac{k \pi}{\alpha}+1, k \in \mathbb{Z}$;

(iii) the case $i=3$ for all $\gamma \neq \frac{(2 k+1) \pi}{2 \alpha}+1, k \in \mathbb{Z}$.

Proof. According to [8, Section 122] the Dirichlet, Neumann, and Zaremba problems in an infinite strip of width $\alpha$ define isomorphisms in Sobolev spaces $H^{s, \delta}\left(\mathbb{R}_{x_{1}} \times \operatorname{int} I_{\alpha}\right):=e^{-x_{1} \delta} H^{s}\left(\operatorname{int} I_{\alpha}\right)$ of smoothness $s \in \mathbb{N}, s \geq 2$, with corresponding exponential weights $\delta$ at $\pm \infty$, except for a discrete set of $\delta \in \mathbb{R}$. The solutions are given by

$$
\begin{aligned}
u_{j, \delta}(x) & =\frac{1}{2 \pi} \int_{-\infty+i \delta}^{\infty+i \delta} e^{i \lambda x_{1}}\left\{\int_{0}^{\alpha} P_{j}\left(\lambda ; x_{2}, y\right) \hat{f}(\lambda, y) d y\right. \\
& \left.+K_{-, j}\left(\lambda ; x_{2}, 0\right) \hat{g}_{-}(\lambda)+K_{+, j}\left(\lambda ; x_{2}, \alpha\right) \hat{g}_{+}(\lambda)\right\} d \lambda
\end{aligned}
$$

for any right sides $f(x) \in H^{s-2, \delta}\left(\mathbb{R} \times \operatorname{int} I_{\alpha}\right), g_{ \pm}\left(x_{1}\right) \in H^{s, \delta}(\mathbb{R})$, where

$$
\begin{aligned}
P_{1}\left(\lambda ; x_{2}, y\right) & :=\frac{\cos i \lambda\left(\left|x_{2}-y\right|-\alpha\right)-\cos i \lambda\left(x_{2}+y-\alpha\right)}{2 i \lambda \sin (i \lambda \alpha)} \\
K_{ \pm, 1}\left(\lambda ; x_{2}, \alpha_{ \pm}\right) & := \pm \partial_{y} P_{1}\left(\lambda ; x_{2}, \alpha_{ \pm}\right) \\
P_{2}\left(\lambda ; x_{2}, y\right) & :=\frac{\cos i \lambda\left(\left|x_{2}-y\right|-\alpha\right)+\cos i \lambda\left(x_{2}+y-\alpha\right)}{2 i \lambda \sin (i \lambda \alpha)} \\
K_{ \pm, 2}\left(\lambda ; x_{2}, \alpha_{ \pm}\right) & := \pm P_{2}\left(\lambda ; x_{2}, \alpha_{ \pm}\right) ; \\
P_{3}\left(\lambda ; x_{2}, y\right) & :=\frac{\sin i \lambda\left(\left|x_{2}-y\right|-\alpha\right)-\sin i \lambda\left(x_{2}+y-\alpha\right)}{2 i \lambda \cos (i \lambda \alpha)} \\
K_{-, 3}\left(\lambda ; x_{2}, 0\right) & :=-\partial_{y} P_{3}\left(\lambda ; x_{2}, y\right) \\
K_{+, 3}\left(\lambda ; x_{2}, \alpha\right) & :=-P_{3}\left(\lambda ; x_{2}, y\right) .
\end{aligned}
$$

Clearly the formula (26) can only be valid when

$$
\begin{aligned}
\delta & \neq \frac{k \pi}{\alpha}, k \in \mathbb{Z} \backslash\{0\} \text { for } j=1, \quad \delta \neq \frac{k \pi}{\alpha}, k \in \mathbb{Z} \text { for } j=2, \\
\delta & \neq \frac{(2 k+1) \pi}{2 \alpha}, k \in \mathbb{Z} \text { for } j=3 .
\end{aligned}
$$

A transformation of the infinite strip to the angle $I_{\alpha}^{\wedge}$, induced by $\mathbb{R}_{x_{1}} \rightarrow$ $\mathbb{R}_{+, r}, r:=e^{-x_{1}}$, transforms the spaces $H^{s, \delta}\left(\operatorname{int} I_{\alpha} \times \mathbb{R}\right)$ to the ones occurring in (22), with $\gamma=\delta+1$. Then we obtain the corresponding isomorphisms for $s \in \mathbb{N}, s \geq 2$, and $\gamma$ as in assertions (i), (ii), and (iii), respectively. Since the operators belong to the cone algebra of boundary value problems, it follows that the isomorphisms (22) are true for arbitrary $s>\frac{3}{2}$, cf. also [7, Section 6.2.1]. 
Remark 2.3 In Theorem 2.2 the infinite stretched cone $I_{\alpha}^{\wedge}$ has a slightly different meaning than in (10). While in (10) we keep in mind the relationship with the 'true' cone $\left(\overline{\mathbb{R}}_{+} \times I_{\alpha}\right) /\left(\{0\} \times I_{\alpha}\right)=K_{\alpha} \cup\{0\}$, with 0 as the tip and conical exit at $\infty$, the cone of Theorem 2.2 is a spindle with two conical points, one at zero and another one at $\infty$. Accordingly, the symbolic structures and ellipticities in both cases are different. In the second case which is simpler there are two conormal symbols, namely, at 0 and $\infty$, respectively. The Fredholm property of (22) is equivalent to the ellipticity of those operators in the cone algebra. The principal symbolic hierarchy on the spindle which is responsible for the ellipticity in this case is a tuple

$$
\sigma(\cdot)=\left(\sigma_{\psi}(\cdot), \sigma_{\partial}(\cdot), \sigma_{\mathrm{c}, 0}(\cdot), \sigma_{\mathrm{c}, \infty}(\cdot)\right)
$$

consisting of interior and boundary symbols as before, moreover, of the conormal symbol $\sigma_{\mathrm{c}, 0}(\cdot)$ at 0 which is equal to $\sigma_{\mathrm{c}}(\cdot)$ from the preceding section, and an analogous conormal symbol $\sigma_{\mathrm{c}, \infty}(\cdot)$ for the conical point at $\infty$.

Using Theorem 2.2 which expresses a special case of Fredholm property and by virtue of the necessity of the ellipticity for the Fredholm property we obtain the following properties.

Corollary 2.4 The conormal symbols

$$
\sigma_{\mathrm{c}, 0}\left(\mathcal{C}_{i}\right)(w): H^{s}\left(\operatorname{int} I_{\alpha}\right) \rightarrow H^{s-2}\left(\operatorname{int} I_{\alpha}\right) \oplus \mathbb{C}^{2},
$$

$s>\frac{3}{2}$, are bijective for all $w \in \Gamma_{1-\gamma}$ and $\gamma$ as in Theorem 2.2 for the corresponding cases $i=1,2,3$. Clearly a similar property is true of $\sigma_{\mathrm{c}, \infty}\left(\mathcal{C}_{i}\right)(w)$.

In fact, we have

$$
\begin{aligned}
& \sigma_{\mathrm{c}, 0}\left(\mathcal{C}_{1}\right)(w)={ }^{\mathrm{t}}\left(w^{2}+\partial_{\phi}^{2} \quad \mathrm{r}_{-} \quad \mathrm{r}_{+}\right), \\
& \sigma_{\mathrm{c}, 0}\left(\mathcal{C}_{2}\right)(w)={ }^{\mathrm{t}}\left(w^{2}+\partial_{\phi}^{2} \quad \mathrm{r}_{-} \partial_{\phi} \quad \mathrm{r}_{+} \partial_{\phi}\right), \\
& \sigma_{\mathrm{c}, 0}\left(\mathcal{C}_{3}\right)(w)={ }^{\mathrm{t}}\left(w^{2}+\partial_{\phi}^{2} \quad \mathrm{r}_{-} \quad \mathrm{r}_{+} \partial_{\phi}\right),
\end{aligned}
$$

where $\mathrm{r}_{ \pm} u:=u\left(\alpha_{ \pm}\right)$for $\alpha_{-}:=0, \alpha_{+}:=\alpha$. The point $w=0$ is a nonbijectivity point only for the Neumann case. For $w=a+i b, w \neq 0$, a simple calculation gives us

$$
\operatorname{ker}\left(w^{2}+\partial_{\phi}^{2}\right)=\left\{c_{1} e^{-b \phi} e^{i a \phi}+c_{2} e^{b \phi} e^{-i a \phi}: c_{1}, c_{2} \in \mathbb{C}\right\} .
$$

Then the boundary conditions yield

$$
e^{-b \alpha} e^{i a \alpha}-e^{b \alpha} e^{-i a \alpha}=0
$$

or, $b=0, a=\frac{k \pi}{\alpha}, k \in \mathbb{Z} \backslash\{0\}$, in the case $i=1,2$, and

$$
e^{-b \alpha} e^{i a \alpha}+e^{b \alpha} e^{-i a \alpha}=0
$$


or, $b=0, a=\frac{(2 k+1) \pi}{2 \alpha}, k \in \mathbb{Z}$ in the case $i=3$.

Recall that

$$
\sigma_{\mathrm{c}}\left(\mathcal{A}_{i}\right)(w)=\sigma_{\mathrm{c}, 0}\left(\mathcal{C}_{i}\right)(w), i=1,2,3 .
$$

The conormal symbols are holomorphic operator functions in $w$, cf. (28), (29), (30).

Theorem 2.5 The points $w \in \mathbb{C}$ where (27) is not bijective are

(i) $w_{k}=\frac{k \pi}{\alpha}, k \in \mathbb{Z} \backslash\{0\}$, for the case $i=1$;

(ii) $w_{k}=\frac{k \pi}{\alpha}, k \in \mathbb{Z}$, for the case $i=2$;

(iii) $w_{k}=\frac{(2 k+1) \pi}{2 \alpha}, k \in \mathbb{Z}$, for the case $i=3$.

All these points are simple (cf. the general notion on meromorphic operator functions of Gohberg and Sigal [4]), except for the point $w_{0}$ in the case (ii) which is a second order zero.

Let us now define the spaces

$$
\mathcal{K}_{i}^{s-2, \gamma-2}\left(I_{\alpha}^{\wedge}\right), i=1,2,3
$$

in a similar manner as (23), (24), (25), by formally replacing $\mathcal{H}$ by $\mathcal{K}$, and let also

$$
\mathcal{K}_{i}^{s-2, \gamma-2 ; g}\left(I_{\alpha}^{\wedge}\right):=\langle r\rangle^{-g} \mathcal{K}_{i}^{s-2, \gamma-2}\left(I_{\alpha}^{\wedge}\right),
$$

$i=1,2,3$, with the multiplication by $\langle r\rangle^{-g}$ in every component.

Theorem 2.6 For every $s>\frac{3}{2}, g \in \mathbb{R}$, the operators

$$
\sigma_{\wedge}\left(\mathcal{A}_{i}\right)(\eta): \mathcal{K}^{s, \gamma ; g}\left(I_{\alpha}^{\wedge}\right) \rightarrow \mathcal{K}_{i}^{s-2, \gamma-2 ; g}\left(I_{\alpha}^{\wedge}\right)
$$

are Fredholm for the same weights as in Theorem 2.2 in the cases $i=1,2,3$.

Proof. By virtue of Theorem 2.1, Corollary 2.4 and relation (31) the result follows for the case $g=0$. However, the exit calculus admits arbitrary power weights at $\infty$; so it suffices to use the fact that ellipticity and Fredholm property are independent of $g$.

We now state the Fredholm indices of (32) for the case $\alpha=\pi$ which is a result from [2] for $i=3$; for the cases $i=1,2$ the values can be obtained by similar methods, see also [7, Section 5.3.4].

Theorem 2.7 For every $s>\frac{3}{2}, g \in \mathbb{R}$, the operators (32) for $\alpha=\pi$ are Fredholm for 
(i) the case $i=1$ for all $\gamma \neq k+1, k \in \mathbb{Z} \backslash\{0\}$, where

$$
\text { ind } \sigma_{\wedge}\left(\mathcal{A}_{1}\right)(\eta)=\left\{\begin{array}{c}
-k \text { for } \gamma \in(k+1, k+2), k \geq 1, \\
-(k+1) \text { for } \gamma \in(k+1, k+2), k \leq-2, \\
0 \text { for } \gamma \in(0,2)
\end{array}\right.
$$

(ii) the case $i=2$ for all $\gamma \neq k, k \in \mathbb{Z}$, where

$$
\text { ind } \sigma_{\wedge}\left(\mathcal{A}_{2}\right)(\eta)=\left\{\begin{array}{c}
-k \text { for } \gamma \in(k+1, k+2), k \geq-1, \\
-(k+1) \text { for } \gamma \in(k+1, k+2), k \leq-2 ;
\end{array}\right.
$$

(iii) the case $i=3$ for all $\gamma \neq k+\frac{1}{2}, k \in \mathbb{Z}$, where

$$
\text { ind } \sigma_{\wedge}\left(\mathcal{A}_{3}\right)(\eta)=-(k+1) \text { for } \gamma \in\left(k+\frac{1}{2}+1, k+\frac{3}{2}+1\right) \text {. }
$$

Let us now interpret Theorem 2.1 as follows. Set

$$
\begin{aligned}
& \gamma_{1 k}(\alpha)=\frac{k \pi}{\alpha}+1, k \in \mathbb{Z} \backslash\{0\}, \quad \gamma_{2 k}(\alpha)=\frac{k \pi}{\alpha}+1, k \in \mathbb{Z}, \\
& \gamma_{3 k}(\alpha)=\frac{(2 k+1) \pi}{2 \alpha}+1, k \in \mathbb{Z},
\end{aligned}
$$

for $0<\alpha \leq 2 \pi$. Let $\sigma_{\wedge}\left(\mathcal{A}_{i}(\alpha, \gamma)\right)(\eta)$ denote the operators (10) for the opening angle $\alpha$ and the weight $\gamma, i=1,2,3$, i.e., we have operators

$$
\sigma_{\wedge}\left(\mathcal{A}_{i}(\alpha, \gamma)\right): \mathcal{K}^{s, \gamma}\left(I_{\alpha}^{\wedge}\right) \rightarrow K_{i}^{s-2, \gamma-2}\left(I_{\alpha}^{\wedge}\right) .
$$

Then Theorem 2.1 tells us that there are integers $\iota_{i k}(\alpha, \gamma)$ such that

$$
\text { ind } \sigma_{\wedge}\left(\mathcal{A}_{i}(\alpha, \gamma)\right)=\iota_{i k}(\alpha, \gamma) \text { for } \gamma_{i k}(\alpha)<\gamma<\gamma_{i(k+1)}(\alpha)
$$

for all $k \in \mathbb{Z} \backslash\{0\}$ for $i=1$ and $k \in \mathbb{Z}$ for $i=2,3$. The numbers $\iota_{i k}(\pi, \gamma)$ are known by Theorem 2.7 .

Now according to Remark 1.2 there are natural families of isomorphisms

$$
\begin{gathered}
L^{s}(\alpha, \gamma): \mathcal{K}^{s, \gamma}\left(I_{\alpha}^{\wedge}\right) \rightarrow \mathcal{K}^{s, \gamma}\left(I_{\pi}^{\wedge}\right), \\
L_{i}^{s-2}(\alpha, \gamma-2): \mathcal{K}_{i}^{s-2, \gamma-2}\left(I_{\alpha}^{\wedge}\right) \rightarrow \mathcal{K}_{i}^{s-2, \gamma-2}\left(I_{\pi}^{\wedge}\right),
\end{gathered}
$$

where we choose the first component of (35) (for the case $s-2, \gamma-2$ ) as (34), and such that $L^{s}(\pi, \gamma)$ and $L_{i}^{s-2}(\pi, \gamma-2)$ are the respective identity maps.

The operators $B_{i}(\alpha, \gamma):=L_{i}^{s-2}(\alpha, \gamma-2) \sigma_{\wedge}\left(\mathcal{A}_{i}(\alpha, \gamma)\right)\left(L^{s}(\alpha, \gamma)\right)^{-1}$ (for any fixed $\eta \neq 0)$ are continuous in $(\alpha, \gamma) \in(0,2 \pi] \times \mathbb{R}$ and Fredholm for the parameters $(\alpha, \gamma)$ in the set

$$
U_{i k}:=\left\{(\alpha, \gamma) \in(0,2 \pi] \times \mathbb{R}: \gamma_{i k}(\alpha)<\gamma<\gamma_{i(k+1)}(\alpha)\right\}
$$


for $k \in \mathbb{Z} \backslash\{0\}$ when $i=1$, and $k \in \mathbb{Z}$ when $i=2,3$. We have

$$
\text { ind } B_{i}(\alpha, \gamma)=\operatorname{ind} \sigma_{\wedge}\left(\mathcal{A}_{i}(\alpha, \gamma)\right)
$$

and, in particular, ind $B_{i}(\pi, \gamma)=$ ind $\sigma_{\wedge}\left(\mathcal{A}_{i}(\pi, \gamma)\right)=\iota_{i k}(\pi, \gamma)$.

Moreover, since the sets $U_{i k}$ are connected for all $i, k$, we have $\iota_{i k}(\alpha, \delta)=$ $\iota_{i k}(\pi, \gamma)$ when $(\alpha, \delta),(\pi, \gamma) \in U_{i k}$.

Using Theorem 2.7 we thus obtain the following results.

Theorem 2.8 For every $s>\frac{3}{2}, g \in \mathbb{R}$, the Fredholm operators (32) have the index

(i) for all $k \in \mathbb{Z} \backslash\{0\}$

$$
\text { ind } \sigma_{\wedge}\left(\mathcal{A}_{1}\right)(\eta)=\left\{\begin{array}{c}
-k \text { for } \gamma \in\left(\frac{k \pi}{\alpha}+1, \frac{(k+1) \pi}{\alpha}+1\right), k \geq 1, \\
-(k+1) \text { for } \gamma \in\left(\frac{k \pi}{\alpha}+1, \frac{(k+1) \pi}{\alpha}+1\right), k \leq-2, \\
0 \text { for } \gamma \in\left(-\frac{\pi}{\alpha}+1, \frac{\pi}{\alpha}+1\right)
\end{array}\right.
$$

(ii) for all $k \in \mathbb{Z}$

$$
\text { ind } \sigma_{\wedge}\left(\mathcal{A}_{2}\right)(\eta)=\left\{\begin{array}{c}
-k \text { for } \gamma \in\left(\frac{k \pi}{\alpha}+1, \frac{(k+1) \pi}{\alpha}+1\right), k \geq-1, \\
-(k+1) \text { for } \gamma \in\left(\frac{k \pi}{\alpha}+1, \frac{(k+1) \pi}{\alpha}+1\right), k \leq-2 ;
\end{array}\right.
$$

(iii) for all $k \in \mathbb{Z}$

$$
\text { ind } \sigma_{\wedge}\left(\mathcal{A}_{3}\right)(\eta)=-(k+1) \text { for } \gamma \in\left(\frac{(2 k+1) \pi}{2 \alpha}+1, \frac{(2 k+3) \pi}{2 \alpha}+1\right) .
$$

\section{$3 \quad$ Elliptic edge conditions and parametrices}

\subsection{Elliptic edge conditions}

Our next objective is to complete the families of Fredholm operators of Theorem 2.6 to families of isomorphisms

$$
\begin{array}{rlll}
\sigma_{\wedge}\left(\mathfrak{A}_{i}\right)(\eta):=\left(\begin{array}{ccc}
\sigma_{\wedge}\left(\mathcal{A}_{i}\right)(\eta) & \sigma_{\wedge}\left(\mathcal{K}_{i}\right)(\eta) \\
\sigma_{\wedge}\left(\mathcal{T}_{i}\right)(\eta) & \sigma_{\wedge}\left(\mathcal{Q}_{i}\right)(\eta)
\end{array}\right) & : & \mathcal{K}^{s, \gamma ; g}\left(I_{\alpha}^{\wedge}\right) \\
& & & \mathbb{C}^{j_{-}} \\
& \rightarrow & \mathcal{K}_{i}^{s-2, \gamma-2 ; g}\left(I_{\alpha}^{\wedge}\right) \\
& & \mathbb{C}^{j_{+}}
\end{array}
$$

$\eta \neq 0$, where $j_{+}-j_{-}=$ind $\sigma_{\wedge}\left(\mathcal{A}_{i}\right)(\eta)$ is known from Theorem 2.8, $j_{ \pm}=$ $j_{ \pm}(i, \gamma), i=1,2,3$, for $\gamma$ in the admitted weight intervals. The process of choosing the so-called trace and potential edge symbols $\sigma_{\wedge}\left(\mathcal{I}_{i}\right)(\eta)$ and 
$\sigma_{\wedge}\left(\mathcal{K}_{i}\right)(\eta)$, respectively, and of the $j_{+} \times j_{-}$matrices of homogeneous functions $\sigma_{\wedge}\left(\mathcal{Q}_{i}\right)(\eta)$ on $T^{*} \mathbb{R}^{q} \backslash\{0\}$ is known for the case $g=0$, see, for instance, [14], [3], [6], or [9]. In the present case for arbitrary $g \in \mathbb{R}$ we want to take care of the group actions in $\mathcal{K}^{s, \gamma ; g}\left(I_{\alpha}^{\wedge}\right)$ and $\mathcal{K}^{s, \gamma ; g}\left(\mathbb{R}_{ \pm}\right)$, respectively, see Example 1.3. We first construct isomorphisms (36) for $|\eta|=1$, smoothly depending on $\eta$ (which is as for $g=0$ ) and then extend them by corresponding twisted homogeneities, associated with the group actions. The orders of homogeneity are completely arbitrary, but we can make a convenient choice.

Writing

$$
\begin{aligned}
\mathcal{W}^{s, \gamma ; g}\left(I_{\alpha}^{\wedge} \times \mathbb{R}^{q}\right):= & \mathcal{W}^{s}\left(\mathbb{R}^{q}, \mathcal{K}^{s, \gamma ; g}\left(I_{\alpha}^{\wedge}\right)\right)_{\kappa^{(1), g}}, \\
\mathcal{W}_{i}^{s-2, \gamma-2 ; g}\left(I_{\alpha}^{\wedge} \times \mathbb{R}^{q}\right) & :=\mathcal{W}^{s-2}\left(\mathbb{R}^{q}, \mathcal{K}^{s-2, \gamma-2 ; g}\left(I_{\alpha}^{\wedge}\right)\right)_{\kappa^{(1), g}} \\
& \oplus \mathcal{W}^{s-\mu_{-}-\frac{1}{2}}\left(\mathbb{R}^{q}, \mathcal{K}^{s-\mu_{-}-\frac{1}{2}, \gamma-\mu_{-}-\frac{1}{2} ; g}\left(\mathbb{R}_{-}\right)\right)_{\kappa^{(0), g}} \\
& \oplus \mathcal{W}^{s-\mu_{+}-\frac{1}{2}}\left(\mathbb{R}^{q}, \mathcal{K}^{s-\mu_{+}-\frac{1}{2}, \gamma-\mu_{+}-\frac{1}{2} ; g}\left(\mathbb{R}_{+}\right)\right)_{\kappa^{(0), g}}
\end{aligned}
$$

where $\mu_{ \pm}=0$ for $i=1, \mu_{ \pm}=1$ for $i=2$, and $\mu_{-}=0, \mu_{+}=1$ for $i=3$, the aim is to associate with (36) a continuous operator

$$
\mathfrak{A}_{i}: \begin{gathered}
\mathcal{W}^{s, \gamma ; g}\left(I_{\alpha}^{\wedge} \times \mathbb{R}^{q}\right) \\
H^{s}\left(\mathbb{R}^{q}, \mathbb{C}^{j_{-}}\right)
\end{gathered} \rightarrow \begin{gathered}
\mathcal{W}_{i}^{s-2, \gamma-2 ; g}\left(I_{\alpha}^{\wedge} \times \mathbb{R}^{q}\right) \\
\rightarrow \\
\end{gathered}
$$

for every $s>\max \left\{\mu_{-}+\frac{1}{2}, \mu_{+}+\frac{1}{2}\right\}$. This determines the choice of the homogeneities. While for the upper left corners we have homogeneities as in Example 1.6, for the other entries we require

$$
\sigma_{\wedge}\left(\mathcal{T}_{i}\right)(\lambda \eta)=\lambda^{2} \sigma_{\wedge}\left(\mathcal{T}_{i}\right)(\eta)\left(\kappa_{\lambda}^{(1), g}\right)^{-1}
$$

and, writing $\sigma_{\wedge}\left(\mathcal{K}_{0, i}\right)(\eta)=\left(\sigma_{\wedge}\left(\mathcal{K}_{i}\right)(\eta), \sigma_{\wedge}\left(\mathcal{K}_{ \pm, i}\right)(\eta)\right)$, according to the three components in the target spaces,

$$
\begin{aligned}
\sigma_{\wedge}\left(\mathcal{K}_{0, i}\right)(\lambda \eta) & =\lambda^{2} \kappa_{\lambda}^{(1), g} \sigma_{\wedge}\left(\mathcal{K}_{0, i}\right)(\eta) \\
\sigma_{\wedge}\left(\mathcal{K}_{ \pm, i}\right)(\lambda \eta) & =\lambda^{\mu_{ \pm}+\frac{1}{2}} \kappa_{\lambda}^{(0), g} \sigma_{\wedge}\left(\mathcal{K}_{ \pm, i}\right)(\eta)
\end{aligned}
$$

for all $\lambda \in \mathbb{R}_{+}$. Knowing $\sigma_{\wedge}\left(\mathcal{I}_{i}\right)(\eta), \sigma_{\wedge}\left(\mathcal{K}_{i}\right)(\eta)$ for $|\eta|=1$, the relations (38), (39), and (40) allow us to define the symbols for all $\eta \neq 0$ by extensions by the corresponding homogeneities. The orders of the entries of $\sigma_{\wedge}\left(\mathcal{Q}_{i}\right)(\eta)$ are 2 in the standard sense. If $\chi(\eta)$ is any excision function we now define

$$
\mathcal{T}_{i}:=\operatorname{Op}\left(\chi \sigma_{\wedge}\left(\mathcal{T}_{i}\right)\right), \quad \mathcal{K}_{i}:=\operatorname{Op}\left(\chi \sigma_{\wedge}\left(\mathcal{K}_{i}\right)\right), \quad \mathcal{Q}_{i}:=\operatorname{Op}\left(\chi \sigma_{\wedge}\left(\mathcal{Q}_{i}\right)\right)
$$

According to Remark 1.7 this gives us continuous operators (37) (see also Proposition 1.8). 


\subsection{Parametrices in weighted edge spaces}

On the (stretched) wedge $I_{\alpha}^{\wedge} \times \mathbb{R}^{q}$ we have a pseudo-differential block matrix algebra of boundary value problems with extra trace and potential data on the edge $\mathbb{R}^{q}$ and for the spaces in (37) for arbitrary $g \in \mathbb{R}$, similarly as in the case $g=0$. The technicalities for $g=0$ may be found, for instance, in [9], see also [7]. An inspection of the details shows that the case of arbitrary $g$ works in a similar manner. In particular, we obtain the following theorem.

Theorem 3.1 For every $s>\frac{3}{2}$ the operator (37) has a parametrix $\mathfrak{P}_{i}$ in the edge algebra of boundary value problems, $i=1,2,3$.

The parametrix $\mathfrak{P}_{i}$ may be found with symbols in the edge calculus of constant coefficients with respect to $y$, and $\mathfrak{P}_{i}$ induces continuous operators in the opposite direction than (37).

The remainders

$$
\mathfrak{I}-\mathfrak{P}_{i} \mathfrak{A}_{i}, \quad \mathfrak{I}-\mathfrak{A}_{i} \mathfrak{P}_{i}
$$

are smoothing in the edge calculus. In particular, when we localise them by composing with functions $\varphi(r, \phi, y), \psi(r, \phi, y) \in C_{0}^{\infty}\left(\overline{\mathbb{R}}_{+} \times I_{\alpha} \times \mathbb{R}^{q}\right)$, they will be compact in the involved spaces.

Corollary 3.2 (Elliptic regularity) $u \in \mathcal{W}^{-\infty, \gamma ; g}\left(I_{\alpha}^{\wedge} \times \mathbb{R}^{q}\right) \oplus H^{-\infty}\left(\mathbb{R}^{q}\right.$, $\left.\mathbb{C}^{j_{-}}\right), \mathfrak{A}_{i} u \in \mathcal{W}_{i}^{s-2, \gamma-2 ; g}\left(I_{\alpha}^{\wedge} \times \mathbb{R}^{q}\right) \oplus H^{s-2}\left(\mathbb{R}^{q}, \mathbb{C}^{j_{+}}\right)$for any $s>\frac{3}{2}$ implies $u \in \mathcal{W}_{i}^{s, \gamma ; g}\left(I_{\alpha}^{\wedge} \times \mathbb{R}^{q}\right) \oplus H^{s}\left(\mathbb{R}^{q}, \mathbb{C}^{j-}\right), i=1,2,3$.

\subsection{Concluding remarks}

The main elements of our results can be carried over to manifolds with edge and boundary, locally modelled on wedges as considered here. Let us replace for the moment $I_{\alpha}$ by any compact $C^{\infty}$ manifold $X$ of dimension $n$ (with or without boundary). Concerning the spaces (16) one has to be careful with the chosen atlas close to the edge. For general $g \in \mathbb{R}$ we impose some conditions. For the invariance it is enough that the transition maps of stretched local wedges $\chi:(r, x, y) \rightarrow(\tilde{r}, \tilde{x}, \tilde{y})$ are independent of $r$ for small $r$.

In general it is required that $\chi$ is the restriction of a diffeomorphism $\chi: \mathbb{R} \times X \times \mathbb{R}^{q} \rightarrow \mathbb{R} \times X \times \mathbb{R}^{q}$ to $\mathbb{R}_{+} \times X \times \mathbb{R}^{q}, \boldsymbol{\chi}(r, x, y)=(\tilde{r}, \tilde{x}, \tilde{y})$, where $\tilde{r}(0, x, y)=0, x \rightarrow \tilde{x}(0, x, y)$ defines a diffeomorphism $X \rightarrow X$, smoothly depending on $y$, and $y \rightarrow \tilde{y}(0, x, y)$ a diffeomorphism $\mathbb{R}^{q} \rightarrow \mathbb{R}^{q}$, independent of $x$. This corresponds to transition maps of wedges $X^{\triangle} \times \mathbb{R}^{q} \rightarrow X^{\triangle} \times$ $\mathbb{R}^{q}, X^{\triangle}=\left(\overline{\mathbb{R}}_{+} \times X\right) /(\{0\} \times X)$ in the category of manifolds with edge, cf. [9, Section 4.1.1]. As noted in Remark 1.4 the spaces for $g=s-\gamma$ have important properties. More precisely, $\mathcal{W}_{\text {loc }}^{s}\left(\mathbb{R}^{q}, \mathcal{K}^{s, \gamma ; g}\left(X^{\wedge}\right)\right)_{\kappa^{g}}$ (which can be defined by locally finite sums of elements of (16) with bounded support 
with respect to $y$ ) are invariant under the transition maps for all $\chi$ of the above-mentioned kind when $g=s-\gamma$.

Boundary value problems on a manifold $M$ with edges are formulated as locally finite sums, with summands coming from operators on wedges, localised by functions from a partition of unity and then pulled back to the manifold itself (in the pseudo-differential case we also add global smoothing operators).

In particular, we can proceed in this way for the Laplacian in $\mathbb{R}^{m}, m=$ $2+q$, restricted to a, say, lense-shaped domain with edge on the boundary of codimension 1 and locally modelled on wedges of opening angle $\alpha$ (in this case it would be natural to assume $0<\alpha \leq \pi$, otherwise, 'lense-shaped' has to be replaced by another notation; $\alpha=2 \pi$ corresponds to a crack situation). Then our problems (together with the extra edge conditions) give rise to Fredholm operators in weighted edge spaces and have parametrices in the edge pseudo-differential calculus. Note that for $\alpha=\pi$ (i.e., when the boundary is smooth and the edge is an interface there) our problems for $i=3$ correspond to the classical Zaremba problem, see [2], while the cases $i=1,2$ correspond to Dirichlet and Neumann data with jumps over the interface.

Other problems, e.g., with jumping oblique derivatives of specific kind (see [5]) can be treated within our approach as well.

Let us finally note that there are also natural subalgebras of edge operators with (discrete and continuous) asymptotics. The singular functions of such asymptotics have a particularly natural shape in the frame of $\mathcal{W}^{s, \gamma ; g_{-}}$ spaces, similarly as is known for $g=0$ from [15]. 
Section 3-i werjum.

In the following we prove the condition (??). Using equation (30) with (??) the left hand side of (??) is equal to

$$
\begin{aligned}
& \frac{c}{2 \pi i} \int_{|\zeta|=1} \frac{\gamma(\zeta)-\bar{\zeta} f(\zeta)}{(\zeta-\bar{\zeta})(1-\bar{z} \zeta)} d \bar{\zeta} \\
- & \frac{1}{\pi} \int_{|\zeta|<1} f(\zeta) P(z, \zeta) d \xi d \eta+\frac{1}{2 \pi i} \int_{|\zeta|=1}(\gamma(\zeta)-\bar{\zeta} f(\zeta)) Q(z, \zeta) d \zeta \\
- & \sum_{k=1}^{\infty} c^{k}(k+1) \frac{1}{\pi} \int_{|\zeta|<1} f(\zeta) \tilde{P}(z, \zeta) d \xi d \eta+\sum_{k=1}^{\infty} c^{k} \frac{1}{2 \pi i} \int_{|\zeta|=1}(\gamma(\zeta)-\bar{\zeta} f(\zeta)) \tilde{Q}(z, \zeta) d \zeta,
\end{aligned}
$$

where

$$
\begin{array}{ccrl}
P(z, \zeta):=\frac{1}{\pi} \int_{|\tilde{\zeta}|<1} \frac{\bar{z}}{(1-\bar{z} \tilde{\zeta})^{2}(\zeta-\tilde{\zeta})^{2}} d \tilde{\xi} d \tilde{\eta}, & Q(z, \zeta):=\frac{1}{\pi} \int_{|\tilde{\zeta}|<1} \frac{\bar{z}}{(1-\bar{z} \tilde{\zeta})^{2}(1-\tilde{\zeta} \bar{\zeta})^{2}\left(\zeta^{2}-c\right)} d \tilde{\xi} d \tilde{\eta}, \\
\tilde{P}(z, \zeta):=\frac{1}{\pi} \int_{|\tilde{\zeta}|<1} \frac{\bar{z}}{(1-\bar{z} \tilde{\zeta})^{2}} \frac{(\zeta-\tilde{\zeta})^{k}}{(\zeta-\tilde{\zeta})^{k+1}} d \tilde{\xi} d \tilde{\eta}, & \tilde{Q}(z, \zeta):=\frac{1}{\pi} \int_{|\tilde{\zeta}|<1} \frac{\bar{z}}{(1-\bar{z} \tilde{\zeta})^{2}} \frac{(\bar{\zeta})^{k}}{(\zeta-\tilde{\zeta})^{k+1}} \frac{d \tilde{\xi} . d \tilde{\eta}}{\zeta-c \tilde{\zeta}}
\end{array}
$$




\section{References}

[1] R. Airapetyan and I. Witt, Propagation of smoothness for edgedegenerate wave equations. In H. Freistühler and G. Warnecke, editors, Eighth International Conference in Magdeburg, February/March 2000, Vol. 140 of Hyperbolic Problems: Theory, Numerics, Applications, pp. 11-18, Basel, 2001, Birkhäuser.

[2] N. Dines, G. Harutyunyan, B.-W. Schulze, The Zaremba problem in edge Sobolev spaces. Preprint 2003/13, Institut für Mathematik, Potsdam, 2003.

[3] Ju.V. Egorov and B.-W. Schulze, Pseudo-differential operators, singularities, applications. Vol. 93 of Operator Theory, Advances and Applicatons. Birkhäuser Verlag, Basel, 1997.

[4] I.C. Gohberg and E.I. Sigal, An operator generalization of the logarithmic residue theorem and the theorem of Rouché. Math. USSR Sbornik, 13 (4): 603-625, 1971.

[5] G. Harutyunyan and B.-W. Schulze, Parametrices of mixed elliptic problems. Math. Nachr., 277: 56-82, 2004.

[6] G. Harutyunyan and B.-W. Schulze, Mixed problems and edge calculus. Rendiconti del Seminario Matematico dell'Universita' e del Politecnico di Torino. (to appear).

[7] G. Harutyunyan and B.-W. Schulze, Elliptic Mixed, Transmission, and Singular Crack Problems. Monograph, European Mathematical Soc. Publ., Zürich. (to appear).

[8] E. Kamke, Differentialgleichungen. Akademische Verlagsgesellschaft 1959.

[9] D. Kapanadze and B.-W. Schulze, Crack theory and edge singularities. Kluwer Academic Publ., Dordrecht, 2003.

[10] V.A. Kondratyev, Boundary value problems for elliptic equations in domains with conical points. Trudy Mosk. Mat. Obshch., 16: 209-292, 1967.

[11] V.A. Kondratyev and O.A. Oleynik, Boundary problems for partial differential equations on non-smooth domains. Uspekhi Mat. Nauk, 38 (2): 3-76, 1983.

[12] V.G. Maz'ja and J. Rossmann, Über die Asymptotik der Lösungen elliptischer Randwertaufgaben in der Umgebung von Kanten. Math. Nachr., 138: 27-53, 1988. 
[13] S. Rempel and B.-W. Schulze, Asymptotics for elliptic mixed boundary problems (pseudo-differential and Mellin operators in spaces with conical singularity). Math. Res., Vol. 50, Akademie-Verlag, Berlin, 1989.

[14] B.-W. Schulze, Pseudo-differential operators on manifolds with edges. In Symp. "Partial Differential Equations", Holzhau 1988, Vol. 112 of Teubner-Texte zur Mathematik, pp. 259-287. Teubner, Leipzig, 1989.

[15] B.-W. Schulze, Pseudo-differential operators on manifolds with singularities. North-Holland, Amsterdam, 1991.

[16] B.-W. Schulze and N.N. Tarkhanov, New algebras of boundary value problems for elliptic pseudodifferential operators. Preprint 2005/08, Institut für Mathematik, Potsdam, 2005. Math. Nachr. (to appear).

[17] J. Seiler, Continuity of edge and corner pseudo-differential operators. Math. Nachr., 205: 163-182, 1999.

[18] N. Tarkhanov, Harmonic integrals on domains with edges. Preprint 2004/20, Institut für Mathematik, Potsdam, 2004. 